\title{
Pharmacometabolomics and Precision Medicine Special Issue Editorial
}

\author{
Nicholas J. W. Rattray ${ }^{1}$ Rima Kaddurah Daouk ${ }^{2}$
}

Published online: 20 March 2017

(C) Springer Science+Business Media New York 2017

The latter portion of the twentieth and the beginning of the twenty-first century has witnessed a "Therapeutic Revolution" in drug treatment, with the development of powerful medicines to treat a broad range of diseases. However, due to disparity in drug response, variation in both efficacy and the occurrence of adverse drug reactions, significant challenges remain in optimizing pharmacologic therapy for individual patients with many remaining resistant to current treatments. The mechanism of action for many drugs remains poorly understood and numerous human diseases express great phenotypical heterogeneity suggesting that these disorders are not unitary conditions but are a collection of entities that are yet to be defined. Thus, this inherent variability coupled with intrinsic differences in pharmacology makes it difficult to predict how an individual patient may respond to a selected therapy driving the need for a more personalized approach to treatment.

To address these issues, newly formed global initiatives have been created to push the agenda for more precise and personalized approaches to treatment. In 2016, the White House announced the Precision Medicine Initiative (PMI) alongside the ambitious 'One Million Person Cohort' to help enable a new era of individualized care through cooperative efforts by researchers, healthcare providers, and

Nicholas J. W. Rattray

nicholas.rattray@yale.edu

Rima Kaddurah Daouk

rima.kaddurahdaouk@duke.edu

1 Department of Environmental Health Sciences, School of Public Health, Yale University, New Haven, CT, USA

2 Department of Psychiatry and Behavioral Medicine, Department of Medicine, Duke Institute for Brain Sciences, Duke University, Durham, NC, USA patients. The National Institutes of Health and its director Francis Collins have called for communities of researchers from around the USA to make the case as to what set of technologies and disciplines would afford the highest level of efficacy in the development of the PMI. Many European and Asian precision medicine initiatives are also emerging, all necessitating the need for scaling up of metabolomics capabilities and standardization of processes.

Our metabolomics community through the Precision Medicine and Pharmacometabolomics Task Group of the Metabolomics Society put forward vision, supporting data and a case for an important role for metabolomics in such precision medicine initiatives. This position is presented in a white paper in this special issue. It is becoming clear that genetics alone cannot explain all variation in drug-response phenotypes and that additional factors, such as environment exposures, diet, age, gender, ethnicity, disease subtype, the use of other medications, and the gut microbiome can all contribute to variation in drug response. New approaches are needed to help capture the effect of these factors to supplement genomic information. The new field of pharmacometabolomics also known as pharmacometabonomics, involves determination of the metabolic state of an individual as affected by environmental, genetic, and gut microbiome influences - the so-called "metabotype"- to define signatures that might inform about treatment outcomes (Kaddurah Daouk and Weinshilboum 2014, 2015; Everett 2015). Pharmacometabolomics also provides tools for mapping the effects of drugs on metabolism and for identifying pathways that contribute to drug-response variation. Baseline information on metabotypes, when combined with signatures for drug exposure, can potentially be used to better define mechanisms of variation in response to drug therapy (Kaddurah-Daouk and Weinshilboum 2014). 
This area of research led by the Pharmacogenomics and Pharmacometabolomics Research Networks (KaddurahDaouk and Weinshilboum 2014, 2015) with significant funding from $\mathrm{NIH}$ has illustrated the promise of using metabolomics data alone, or in combination with genomics data to better inform clinicians about treatment outcomes and mechanisms that underlie variation in response to treatments. The White Paper in this special issue captures developments in this new and rapidly growing field with exemplification from over ten commonly used drugs. Sex and ethnic background contribution to variation of response can be captured and explained in part by metabolome data. A historic study in animals by Imperial researchers and their pharmaceutical consortium revealed how metabolomics data at base line can inform about drug metabolism and toxicity and infer the role of the gut microbiome (Clayton et al. 2006). The first illustrated concept of "pharmacometabolomics informing pharmacogenomics" (Ji et al. 2011) led the way for using an integrated metabolic genetic approach to drug response phenotyping.

Vast number of studies has subsequently validated the importance of using metabolomics data in precision medicine initiatives. With such unprecedented numbers of samples, standardization at every level becomes key. One approach of large-scale metabolic assessment has already been set in to action in the UK residing at Imperial College London with a growing number of linked National Phenome Centers. These have been designed to harmonize the collection and analytical protocols used in metabolic phenotyping and aim to impact healthcare similarly by creating multilayered understanding of disease process and environmental interaction. The Phenome concept has also spread globally with a network of international partners that share an interest in building a global infrastructure around harmonized research methods and technologies.

In this special issue we take pleasure in presenting compelling evidence for the important role for metabolomics in informing about drug response, drug mechanism of action and molecular basis for variation in response. Through a series of reviews and original articles the authors of this special issue highlight several applications for pharmacometabolomics in the study of drug effects. They illustrate its utility in clinical pharmacology and underline the importance of the emerging field of quantitative and systems pharmacology where large data is starting to redefine our understanding of how drugs work and work differently.

Armitage and Southam discuss how metabolomics and lipidomics data can be used to identify metabolic biomarkers of cancer and to understand the mechanism-of-action of anticancer therapies. They present considerations that can maximize the clinical value of metabolic cancer biomarkers including case-control, prognostic and longitudinal study designs.
The significance of variation in response to commonly used antihypertensive therapies is investigated by de Oliveira et al. They exemplify how the beta-blocker atenolol, which is known to cause a therapeutic benefit that differs based on ethnic background, is associated with adverse events including hyperglycemia and incident diabetes that may offset the benefits of blood pressure reduction. By combining large untargeted metabolomic and genomic datasets acquired from hypertensive individuals treated with atenolol, they illustrate how it may be possible to better understand the pathways that most impact the therapeutic benefit of the development of an adverse glycemic state and how this can eventually lead to predictive biomarkers that can optimize use of antihypertensives among populations. Weng et al. also focus on the metabolic side effects of atenolol in a pharmacogenomic responses study in which they target acylcarnitines implicated in mitochondrial energetics. The level of these metabolites in serum are shown to inform about adverse cardiometabolic responses including glucose changes and diabetes development.

Broad off target metabolic effects of drug response are also discussed by Cowan et al. in which they apply metabolomics tools to study the controversial gastrointestinal peptide obestatin. They demonstrate that treatment with the drug affects phospholipid turnover and influences lipid homeostasis, whilst providing convincing evidence that obestatin may be acting to ameliorate diet-induced impairments in lipid metabolism, and it may influence steroid, bile acid, PAF and glutathione metabolism.

Statins are another widely prescribed drug class used for the prevention and treatment of cardiovascular disease. They reduce low density lipoprotein cholesterol (LDL-C) levels by inhibiting their biosynthesis. Despite providing major benefits, statins are associated with adverse effects that including muscle myopathy as well as development of type II diabetes mellitus (T2DM). In sub populations of patients this may result in premature discontinuation of treatment and there are no reliable biomarkers for predicting clinical side effects in vulnerable individuals. Elbadawi-Sidhu et al. present supporting data that pharmacometabolomics provides powerful tools for identifying global biochemical changes induced by statin treatment, providing mechanistic insights about drug mechanism of action, development of side effects and related biomarkers.

With the rise of obesity, diabetes and stressed linked disease often been seen as associated with western life-style, it is vital that metabolomics research is also applied to tropical and infectious diseases that often infect and kill far more people. Stoessel and co-authors apply metabolomics to the study of investigational drug for treatment of African trypanosomiasis (HAT). Trypanosoma brucei is the causative agent of HAT, which is responsible for tens of thousands of deaths every year. 
The anti-trypanosomal compound, 3-(oxazolo[4,5- $b$ ] pyridine-2-yl)anilide (OXPA), was initially identified in a phenotypic screen and subsequently optimized by structure-activity directed medicinal chemistry. It has been shown to be non-toxic and to be active against a number of trypanosomatid parasites. However, nothing is known about its mechanism of action. The authors use an untargeted metabolomics approach to investigate the biochemical effects and potential mode of action of this compound in $T$. brucei and highlight an important role for ceramide metabolism and sphingolipid pathway as a promising drug target in treatment.

Once administered, drug side effects are a vital consideration in the viability of a drug making it to market and Beger and Flynn review the application of metabolomics to this important topic. They discuss how pharmaocometabolomics work relevant to drug safety including factors besides genetics can play a role in how a subject responds to a drug treatment. Pharmacometabolomics studies on drug-induced liver toxicity, the use of pharmacometabolomics to detect and predict drug interactions, and future applications of pharmacometabolomics in drug safety are discussed.

Lin et al. also evaluate drug clearance by investigating the cancer drug busulfan and illustrate how metabolomics data can be used in a predictive model to inform about its clearance profile. These results reinforce the potential of pharmacometabolomics as a critical tool in personalized medicine, with the potential to improve the personalized dosing of drugs with a narrow therapeutic index such as busulfan.

With metabolomics being very much a data driven science, no special issue in the area would be complete without major considerations as to how technology and statistical tools alongside data integration contribute to the field. A review and series of papers address how metabolomics data can inform about not only pharmacodynamic profile of medications but also their pharmacokinetic profiles. The uptake of drugs, their metabolism and transport to site of action can all be influenced by metabolic states and regulation of gut metabolism. Kanta et al. review the promise of using pharmacometabolomics data to inform pharmacokinetics in efforts for more personalized therapy. Van der Hooft et al. also use innovative approaches to study and visualize antihypertensive drug metabolites in untargeted metabolomics experiments based on the spectral similarity of their fragmentation spectra. A molecular networking approach was applied to seek fragmentation spectra from urine derived from antihypertensive drug metabolites. This methodology offers unprecedented capability in the untargeted identification of drugs and their metabolites at the population level and has great potential to contribute to understanding stratified responses to drugs where differences in drug metabolism may determine treatment outcome.

The use of isotope labeling is also another hot topic used to investigate mode of action and Lane et al. address the important topic related deeper understanding of effects of drugs on metabolism and for variation in response to treatment. The "Stable Isotope Resolved Metabolomics (SIRM)" approach is exemplified from studies in cancer therapy. Their review compares the advantages and disadvantages of different model biological systems for determining the metabolic functions of cells in complex environments and how they may change in different disease states, respond to therapeutic interventions and how stable isotope use can transform our molecular understanding.

Neavin et al. also review recent developments in the rapidly growing field of pharmacmetabolomics informing pharmacogenomics. They outline the challenges and opportunities associated with studies in which metabolomic data have been merged with genomics in an attempt to gain novel insight into mechanisms associated with variation in drug response phenotypes.

We feel that opinions within the clinical community regarding the use and applicability of metabolomics tools to address the grand challenges of drug mode of action, efficacy and side effects are changing. More and more medical researchers are being empowered by the tools and information that metabolomics offers and are beginning to understand that analyzing an actual expressed phenotype is essential in all aspects of drug development, the understanding of disease heterogeneity, variation of response and hence all precision medicine initiatives.

\section{References}

Armitage, E. G., \& Southam, A. D. (2016). Monitoring cancer prognosis, diagnosis and treatment efficacy using metabolomics and lipidomics. Metabolomics, 12, 146.

Beger, R. D., Dunn, W., Schmidt, M. A., Gross, S. S., Kirwan, J. A., Cascante, M., Brennan, L., Wishart, D. S., Oresic, M., Hankemeier, T., Broadhurst, D. I., Lane, A. N., Suhre, K., Kastenmüller, G., Sumner, S. J., Thiele, I., Fiehn, O., \& Kaddurah-Daouk, R. "Precision Medicine and Pharmacometabolomics Task Group" - Metabolomics Society Initiative. (2016). Metabolomics enables precision medicine: "A white paper, community perspective". Metabolomics 12: 149.

Beger, R. D., \& Flynn, T. J. (2016). Pharmacometabolomics in drug safety and drug-exposome interactions. Metabolomics, 12, 123.

Clayton, T. A., Lindon, J. C., Cloarec, O., Antti, H., Charuel, C., Hanton, G., et al. (2006). Pharmaco-metabonomic phenotyping and personalized drug treatment. Nature, 440(7087), 1073-1077.

Cowan, E., Kumar, P., Burch, K. J., Grieve, D. J., Green, B. D., \& Graham, S. F. (2016). Treatment of lean and diet-induced obesity (DIO) mice with a novel stable obestatin analogue alters plasma metabolite levels as detected by untargeted LC-MS metabolomics. Metabolomics, 12, 124. 
de Oliveira, F. A., Shahin, M. H., Gong, Y., McDonough, C. W., Beitelshees, A. L., Gums, J. G., Chapman, A. B., Boerwinkle, E., Turner, S. T., Frye, R. F., Fiehn, O., Kaddurah-Daouk, R., Johnson, J. A., \& Cooper-DeHoff, R. M. (2016). Novel plasma biomarker of atenolol-induced hyperglycemia identified through a metabolomics-genomics integrative approach. Metabolomics, $12,129$.

Elbadawi-Sidhu, M., Baillie, R. A., Zhu, H., Chen, Y-D.I., Goodarzi, M. O., Rotter, J. I., Krauss, R. M., Fiehn, O., \& KaddurahDaouk, R. (2017). Pharmacometabolomic signature links simvastatin therapy and insulin resistance. Metabolomics, 13, 11 .

Everett, J. R. (2015). Pharmacometabolomics in humans: a new tool for personalized medicine. Pharmacogenomics, 16(7), 737-754.

Ji, Y., Hebbring, S., Zhu, H., Jenkins, G. D., Biernacka, J., Snyder, K., Drews, M., Fiehn, O., Zeng, Z., Schaid, D., Mrazek, D. A., Kaddurah-Daouk, R., \& Weinshilboum, R. M. (2011). Glycine and a glycine dehydrogenase (GLDC) SNP as citalopram/escitalopram response biomarkers in depression: pharmacometabolomicsinformed pharmacogenomics. Clinical pharmacology and therapeutics, 89(1), 97-104.

Kaddurah-Daouk, R., \& Weinshilboum, R. Pharmacometabolomics Research Network. (2015). Metabolomic signatures for drug response phenotypes-pharmacometabolomics enables precision medicine. Clinical pharmacology and therapeutics, 98(1):71-75.

Kaddurah-Daouk, R., \& Weinshilboum, R. M. Pharmacometabolomics Research Network. (2014). Pharmacometabolomics: implications for clinical pharmacology and systems pharmacology. Clinical pharmacology and therapeutics, 95(2), 154-167.

Kantae, V., Krekels, E. H. J., Van Esdonk, M. J., Lindenburg, P., Harms, A. C., Knibbe, C. A. J., Van der Graaf, P. H., \& Hankemeier, T. (2017). Integration of pharmacometabolomics with pharmacokinetics and pharmacodynamics: Towards personalized drug therapy. Metabolomics, 13, 9 .

Lane, A. N., Higashi, R. M., \& Fan, T. W. -M. (2016). Preclinical models for interrogating drug action in human cancers using Stable Isotope Resolved Metabolomics (SIRM). Metabolomics, 12, 118.

Lin, Y. S., Kerr, S. J., Randolph, T., Shireman, L. M., Senn, T., \& McCune, J. S. (2016). Prediction of intravenous busulfan clearance by endogenous plasma biomarkers using global pharmacometabolomics. Metabolomics, 12, 161.

Neavin, D., Kaddurah-Daouk, R., \& Weinshilboum, R. (2016). Pharmacometabolomics informs pharmacogenomics. Metabolomics, $12,121$.

Stoessel, D., Nowell, C. J., Jones, A. J., Ferrins, L., Ellis, K. M., Riley, J., Rahmani, R., Read, K. D., McConville, M. J., Avery, V. M., Baell, J. B., \& Creek, D. J. (2016). Metabolomics and lipidomics reveal perturbation of sphingolipid metabolism by a novel anti-trypanosomal 3-(oxazolo[4,5- $b]$ pyridine-2-yl)anilide. Metabolomics, 12, 126.

van der Hooft, J. J. J., Padmanabhan, S., Burgess, K. E. V., \& Barrett, M. P. (2016). Urinary antihypertensive drug metabolite screening using molecular networking coupled to high-resolution mass spectrometry fragmentation. Metabolomics, 12, 125.

Weng, L., Gong, Y., Culver, J., Gardell, S. J., Petucci, C., Morse, A. M., Frye, R. F., Turner, S. T., Chapman, A., Boerwinkle, E., Gums, J., Beitelshees, A. L., Borum, P. R., Johnson, J. A., Garrett, T. J., McIntyre, L. M., \& Cooper-DeHoff, R. M. (2016). Presence of arachidonoyl-carnitine is associated with adverse cardiometabolic responses in hypertensive patients treated with atenolol. Metabolomics, 12, 160. 